\title{
Gestión del conocimiento en instituciones universitarias
}

\author{
Juan Antonio Pastor Sánchez \\ Servicio de Información \\ Universidad de Murcia
}

\begin{abstract}
Creo que contemplamos las pautas reales desde un punto de vista restringido. Y ese punto de vista restringido dice que la gente hace cosas deliberadas, en complicidad, dirigidas contra uno, cuando en realidad existen pautas que no dependen de las personas. Y no están dirigidas contra ninguno de nosotros; son mucho más amplias, y funcionan gracias a todos.
\end{abstract}

Philip K. Dick

\subsection{Resumen}

Se estudia el servicio de información universitario (SIU) en el marco de la política de gestión del conocimiento de las universidades. Partiendo del análisis de la estructura universitaria y de sus necesidades y flujos informativos, se definen los objetivos del SIU y sus diferentes posibilidades organizativas. Se consideran las tecnologías disponibles, principalmente las que aseguran el trabajo en grupo sobre una arquitectura cliente-servidor con interfaz web. Finalmente, se describen los productos fundamentales elaborados por un SIU, entre los que se destaca la utilización de la tecnología WWW para la elaboración de portales universitarios, verdadero eje de la organización y difusión de la información en las universidades.

Palabras clave: Servicios de información universitarios. Gestión del conocimiento.

\subsection{Abstract}

The university information services (UIS) are studied in the context of the knowledge management policies of universities. From an analysis of the structure of universities and their information needs and flows, UIS aims are defined and the different managerial approaches are sketched. The technological infrastructure is envisioned as a groupware environment on a web front-ended client-ser- 
ver architecture. Finally, the main informative products of a UIS are described, emphasising the use of WWW technology for building corporative portals, evaluated as the core of the organisation and sharing of knowledge in universities.

Keywords: Universitary information systems. Knowledge management.

\section{Introducción}

Los cambios producidos a finales del siglo XX afectan a todos los ámbitos de la vida. Se ha producido un proceso de democratización ideológica en los medios de comunicación. Sin embargo, a partir de la aparición de Internet y su difusión entre los consumidores de información, hemos asistido a un proceso de participación cualitativamente diferente. Los usuarios ya no se limitan a consultar información, también tienen un canal para hacer públicas sus ideas, opiniones y conocimientos. Además, esta participación también se centra en la obtención de información de forma personalizada, acorde a las necesidades de información y a la posibilidad de comunicación interactiva entre usuarios y gestores de los sistemas y servicios de información.

Las universidades españolas han contribuido al uso generalizado de las fuentes de información en Internet. Este consumo de información se ha canalizado a través del uso de servicios de todo tipo (comerciales, administrativos, educativos, etc.). En un principio, la difusión de las redes basadas en TCP/IP y el uso de protocolos de Internet de forma experimental o interna originaron la adopción y selección natural de aplicaciones para el proceso de almacenamiento, recuperación y difusión de información a través de Internet.

Una de las causas del crecimiento de las redes de ordenadores se debe al incremento del volumen de negocio y a la necesidad de procesos de cambio, comunicación, conectividad, cooperación y coordinación. Sin embargo, la existencia de dichas redes y por tanto de la tecnología disponible en el mercado no garantiza que dichos recursos (hardware, software y netware) se utilicen de un modo eficaz y, por tanto, ofrezca el máximo beneficio de su aplicación dentro de cualquier ámbito, incluido el ámbito universitario, donde el concepto de "beneficio" no se aplica solamente al beneficio académico, sino a la asimilación de conocimientos, mejora de las herramientas docentes, obtención de resultados de investigación y optimización en el proceso de gestión.

Actualmente nos encontramos en un momento clave para el desarrollo de las instituciones universitarias en todos sus aspectos. La aplicación de las tecnologías de la información debe tenerse en cuenta, en los procesos de gestión y como parte de los contenidos docentes, así como para conseguir una gestión del conocimiento para la toma de decisiones y como modelo para organizar, tratar, difundir información e intercambiar información de un modo ordenado e integrado 
(Cornellá, 1999). En definitiva, no hay que considerar a las tecnologías de la información como un fin en si mismas, sino como una herramienta aplicada a la Gestión de Conocimiento.

La idea clásica de un servicio de información dirigido principalmente a los alumnos debe ser superada: hay que tener en cuenta a toda la Comunidad Universitaria (Aalumnos, profesores, investigadores y personal de administración y servicios). Incluso pensar que un Servicio de Información Universitario orientado a la elaboración de páginas WEB y publicaciones también debe ampliarse mediante un modelo que nos permita la organización interna de funciones y tareas para crear unos productos y servicios adaptables a las necesidades de información de cada usuario.

\section{Objetivo: ¿La Gestión del Conocimiento?}

Cuando hablamos de gestión del conocimiento o de gestores del conocimiento hay tener en cuenta la estructura que subyace a todo ello. La realidad se conforma según la tratamos a través de nuestros sentidos. Nuestro universo, el universo de cada persona, tiene como núcleo fundamental la información percibida y adquirida que conforma la realidad del individuo. Los hechos y datos (al igual que la materia) se generan y se estructuran según unas reglas. El Cosmos se constituye en todos sus aspectos según dichas reglas, de forma que nuestro ADN, las leyes físicas o la estructura de la materia siguen dichas leyes.

El caos supone el desconocimiento, la ignorancia de dichas leyes. Los hechos que se intentan explicar a través de la Teoría del Caos tienen tantos factores y reglas que se escapan a nuestros sentidos. Es fundamental distinguir la información (hechos percibidos de forma estructurada y aislados de nuestras experiencias) del conocimiento (información integrada en nuestras experiencias y esquemas mentales). Por ello, lo que no podemos estructurar debido a su complejidad se denomina caos (Pastor Sánchez, 1996).

En realidad, la información es la causa primera del conocimiento a través de una serie de procesos (percepción, análisis, evaluación). El conocimiento es algo personal y subjetivo donde influyen las experiencias pasadas, presentes e incluso las expectativas personales del individuo (Currás, 1995). Aquí es donde entra en juego la Teoría de la Relatividad. Albert Einstein afirmaba que pese a existir unas reglas generales de comportamiento para cualquier hecho a estudiar, las conclusiones y resultados dependen del observador.

Pero entonces, nosotros, como documentalistas, bibliotecarios, archiveros, museólogos, gestores de información, etc. ¿podemos gestionar conocimiento? En mi opinión la respuesta es clara: no. Realmente lo que el profesional de la información puede hacer es ayudar al usuario final a gestionar su conocimiento

Scire. $6: 2$ (jul.-dic. 2000) 99-120. 
personal. Nuestra tarea es la de diseñar y desarrollar herramientas, métodos, sistemas y servicios que posibiliten al usuario compartir, transmitir y difundir su conocimiento, al tiempo que le ayuden en la adquisición de información para una asimilación e integración de la misma y una posterior generación de conocimiento (Arboníes, 2000). Thomas Davenport define la Gestión del Conocimiento como "el proceso sistemático de encontrar, seleccionar, organizar, destilar y presentar la información de una manera que mejore la comprensión de un área específica de interés para los miembros de una organización" (Davenport, 1996). Según Eduardo Bueno Campos, gestión del conocimiento es "la función que planifica, coordina y controla los flujos de conocimientos que se producen en la empresa en relación con sus actividades y con su entorno con el fin de crear unas competencias esenciales" (Bueno Campos, 1998).

Por lo tanto, hablar de gestión del conocimiento y plantear nuestras hipótesis y actividades de trabajo desde el punto de vista del conocimiento (y no de la información) nos acerca al individuo, por lo que tratar la información a través de una serie de procesos y herramientas no pasa de ser un medio y no un fin en sí mismo. Pero la gestión de la información documental constituye un puente hacia nuestro objeto, y el puente debe ser construido, modernizado, pasar de la madera, a la piedra y de esta al acero. Diseñar sistemas y servicios de información que simplemente tengan como objetivo el estar bien diseñados o cumplir determinadas especificaciones sin tener en cuenta las necesidades del usuario final constituye uno de los errores más frecuentes en nuestro trabajo y que deben evitarse a toda costa.

Hoy día hay que hablar de sistemas de información cooperativos en donde una serie de individuos crean, mantienen y consultan un conjunto de información que es susceptible de ser utilizado por la totalidad de la organización en la que se encuentra dicho sistema. Los conceptos de red y de individuo están en equilibrio: el usuario puede disponer de documentos privados y personales y al mismo tiempo hacer accesibles otros a un conjunto de personas. A nivel práctico pasar de la gestión de información a la gestión de conocimiento conlleva un cambio de mentalidad en los trabajadores de una organización y aplicar una serie de procesos de reingeniería metodológicos y tecnológicos (Martínez Méndez, 1999), como ya veremos más adelante.

El conocimiento no surge con la acumulación de información sin más, sino que se nos muestra cuando la información se estructura de forma agregada. Por ello el conocimiento se encuentra repartido entre las mentes de todos los miembros de la comunidad universitaria y entre los sistemas de información de la misma (Saorín, 1997). 


\section{Bases para la creación de un Entorno de Trabajo}

\subsection{Las Instituciones Universitarias}

La creación y aplicación de un modelo de Gestión del Conocimiento en una universidad debe partir de la necesidad de alcanzar a cualquier universitario, sea profesor, alumno, investigador, auxiliar administrativo o becario. Los servicios orientados a un sólo tipo de usuarios tienden a encerrarse en si mismos, a solapar tareas que ya realizan otras unidades o dejar desiertas ciertas actividades muy necesarias.

La propia estructura de la organización determina el flujo informativo, y por tanto, es conveniente recordar que la evolución y desarrollo de las distintas estructuras ha ido imponiendo en las organizaciones los canales más apropiados y eficaces para apoyar la comunicación entre personas y grupos. A medida que se produzcan cambios sociales, y especialmente culturales, se darán variaciones en la estructura organizativa. Si la estructura permite una gran autonomía o flexibilidad a sus partes constituyentes se generará un tipo de flujo muy diferente al que se da en una estructura muy jerarquizada, y esto va a influir en el diseño del sistema de información: Habrá que analizar el tipo de información que se genera y lo que se necesita, pero también quien la usa y como o para qué.

Por regla general (salvando excepciones de cargos y denominación) la estructura orgánica de las universidades españolas se centra en los siguientes órganos y unidades:

\section{1. Órganos de Gobierno}

a. Rector/Presidente: Suele ser la máxima autoridad de la Universidad

b. Equipo rectoral/Equipo de dirección: Suele estar formado por Vicerrectores/Consejeros. Cada miembro de dicho equipo abarca un área de gestión.

c. Gerencia: Su ámbito de actuación es la gestión de los servicios económicos, administrativos y de recursos humanos de la Universidad.

d. Secretaría general: Sus funciones es la asistencia al Rector en las tareas de organización y administración de la Universidad así como constituirse como fedatario de los actos y acuerdos que se tomen en la Junta de Gobierno.

e. Coordinadores: Son los interlocutores entre el rector, los miembros del equipo rectoral, gerencia y secretaría general y los servicios universitarios.

f. Claustro universitario: Máximo órgano representativo de todos los miembros de la Comunidad Universitaria. 
g. Junta de gobierno: Es el órgano de gobierno ordinario de la Universidad

h. Consejo social: Es el órgano de participación de la sociedad en la Universidad, para la supervisión de actividades de carácter económico de la misma y del rendimiento de sus servicios, así como para promover la colaboración de la sociedad en la financiación de la universidad.

i. Juntas de centro: Su objetivo es la representación y gobierno ordinario de los centros universitarios.

j. Consejos de departamento: Es el órgano de representación y dirección de los departamentos universitarios.

2. Centros: Encargados de la gestión administrativa y de la organización de las enseñanzas conducentes a la obtención de los correspondientes títulos académicos.

3. Departamentos: Son órganos básicos encargados de organizar y desarrollar la investigación y las enseñanzas propias de su área o áreas de conocimiento en una o varias.

4. Institutos universitarios y escuelas profesionales: Tienen como funciones la investigación, la enseñanza especializada, la creación artística, la actualización y especialización profesional de los titulados universitarios y el control, asesoramiento y actuación técnica en el ámbito de su competencia.

\begin{tabular}{|c|c|c|}
\hline Estudiantes & $\begin{array}{c}\text { Personal } \\
\text { de administración } \\
\text { y servicios }\end{array}$ & $\begin{array}{c}\text { Personal } \\
\text { docente e } \\
\text { investigador }\end{array}$ \\
\hline $\begin{array}{c}\text { Información académica } \\
\text { Contenidos docentes } \\
\text { Servicios universitarios } \\
\text { Becas y ayudas } \\
\text { Premios y concursos } \\
\text { Formación extracurricular } \\
\text { Actividades de ocio } \\
\text { Empleo } \\
\text { Etc. }\end{array}$ & $\begin{array}{c}\text { Procedimientos } \\
\text { administrativos } \\
\text { Información laboral } \\
\text { Ayudas sociales } \\
\text { Formación } \\
\text { Empleo } \\
\text { Servicios universitarios } \\
\text { Etc. }\end{array}$ & $\begin{array}{c}\text { Organización docente } \\
\text { Convocatorias de } \\
\text { investigación } \\
\text { Información bibliográfica } \\
\text { Acuerdos y resoluciones } \\
\text { Formación de personal } \\
\text { investigador } \\
\text { Actividades de Investigación } \\
\text { Ayudas sociales } \\
\text { Etc. }\end{array}$ \\
\hline
\end{tabular}

Cuadro 1. Necesidades de Información

Scire. $6: 2$ (jul.-dic. 2000) 99-120. 
5. Servicios universitarios: Unidades con organización propia que sirven de apoyo a la docencia, investigación y gestión de la Universidad, y al desarrollo social y cultural de la comunidad universitaria.

La comunidad universitaria está compuesta por personal docente e investigador, personal de administración y servicios, y por supuesto, el colectivo más importante y numeroso: los estudiantes. No hay que olvidar tampoco que en cualquier momento, cualquier persona (miembro o no de la universidad) puede convertirse en usuario de un servicio de información universitario.

\subsection{Estructura y contenidos informativos $=$ Necesidades de Información}

Uno de los puntos más importantes a la hora de crear un sistema de información es decidir qué información va a gestionar. En este se gestionará información del ámbito de la universidad en la que opere el sistema, información de otras universidades y recursos, y fuentes de información sobre actividades y áreas sociales de interés. Lo cierto es que cada tipo de usuario tiene unas necesidades concretas de información. En el cuadro 1 se presenta un resumen de dichas necesidades.

El tratamiento que debe recibir la información que se procesa en el sistema debe amoldarse a los usuarios hacia los que va dirigida. Seguimos trabajando con multitud de documentos en papel. Por lo tanto, el sistema debe estar orientado tanto al procesamiento de información electrónica como al de la información en soporte papel (aplicando técnicas de archivo y OCR). Es conveniente el uso de bases de datos — bien referenciales o en texto completo-. En cualquier caso la aplicación de técnicas de resumen facilitará el trabajo de gestión enormemente. Las herramientas del sistema de información servirán para la creación de productos de información o bien serán usadas directamente por el propio usuario.

\subsection{Organización de un Servicio de Información Universitario}

Se hace patente la necesidad de un servicio de información universitario (SIU) que puede definirse como: Un servicio dirigido a la totalidad de la comunidad universitaria y orientado a la coordinación de tareas desarrolladas por una serie de puntos de información periféricos y centrales, en los que se llevan a cabo trabajos de obtención, tratamiento, acceso y recuperación de la información de un modo sistemático y homogéneo. Además, también podrá desarrollar actividades de asesoría en gestión documental para otras unidades de la Comunidad Universitaria e incluso fuera de ella.

Los nuevos tiempos requieren nuevas estructuras de organización del trabajo y nuevos profesionales. Obviamente necesitaremos a un profesional capaz de participar de forma activa y dinámica en el diseño, desarrollo e implementación de los sistemas de información (Martínez Méndez, 1997). Evidentemente nos estamos refiriendo a personas con formación universitaria en el área de Gestión

Scire. $6: 2$ (jul.-dic. 2000) 99-120. 
de Información y Documentación y con amplios (que no profundos) conocimientos de sistemas informáticos. Esta combinación de conocimientos de gestión, técnicos y metodológicos nos permitirá contar con la garantía de profesionales preparados, no solamente para comenzar a trabajar en este campo, sino con capacidades de aprendizaje para estar siempre al día en el campo de las últimas tendencias tecnológicas y metodológicas y con la capacidad de formar y fomentar el uso de sistemas de información entre los usuarios finales (Sebastiá, 1992).

La estructura orgánica de este servicio deberá contemplar la realidad del entorno que le rodea. Básicamente podría seguir un modelo compuesto por una Unidad de Gestión de Información y una Unidad de Atención al Público. La Unidad de Gestión de Información deberá realizar tareas de gestión, elaboración, y difusión de información de cualquier tipo que pueda interesarle a los miembros de la comunidad universitaria. Por su parte la Unidad de Atención al Público atendería a los usuarios de un modo personalizado, orientándolos en las fuentes a consultar, suministrándole información específica a una necesidad concreta, instruyendo a los usuarios en el manejo de los sistemas de información y difundiendo las actividades de todo el servicio.

Respecto a la organización y reparto de tareas entre el personal de propio servicio existen cuatro opciones:

1. Contenidos temáticos: Cada gestor se encarga de abarcar un área temática según el contenido de la información documental que entra en el sistema.

2. Programas de actividades: Las actividades del servicio de información se estructuran en programas y cada uno de ellos se asignados a un gestor determinado que a partir de ese momento es responsable de su correcto funcionamiento.

3. Áreas de trabajo especializadas: Este esquema establece áreas especializadas de reparto de actividades. A grandes rasgos se establecen cuatro áreas especializadas (Fig. 1)

a. Gestión de información: Centraliza los flujos de entrada de información. También realiza su tratamiento y gestiona bases de datos documentales.

b. Sistemas de información multimedia: Elabora y diseña herramientas multimedia para la consulta y difusión de información, principalmente a través del Web y publicaciones electrónicas.

c. Elaboración de publicaciones: Elaborará publicaciones en soporte papel del tipo de guías, boletines, folletos, etc.

d. Atención al público.

En este esquema el Área de Gestión de Información constituye una base de conocimiento para el resto de áreas del servicio de información. Lo más reco- 


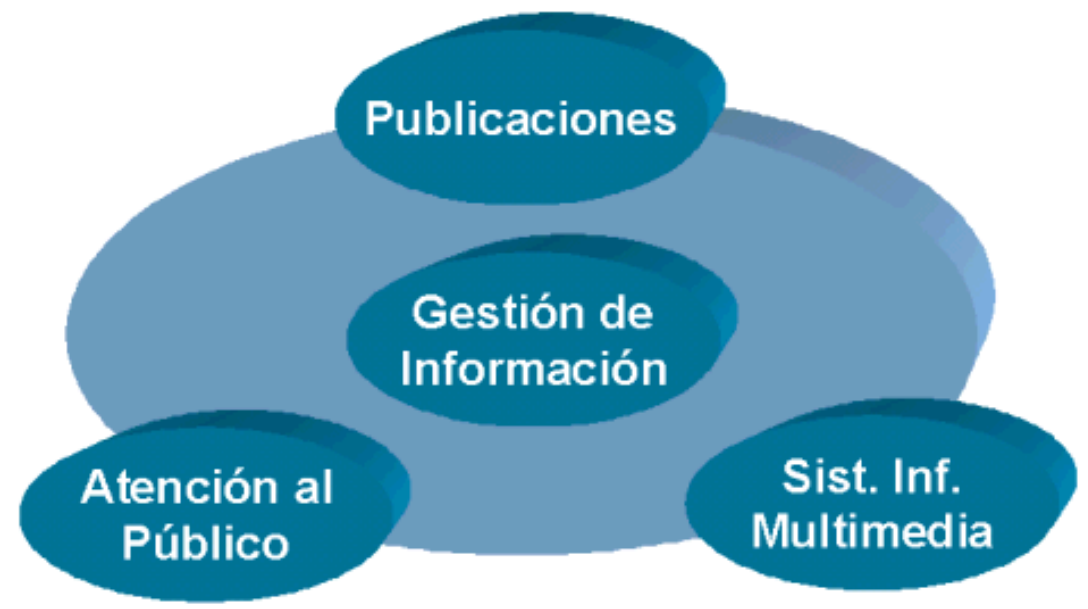

Figura 1. Organización del trabajo en áreas especializadas

mendable es establecer un esquema mixto organizando el trabajo en programas concretos y asignándolo a áreas de trabajo. Es evidente que la solución que se adopte dependerá en gran medida de los recursos humanos y competencias del servicio de información.

\subsection{Integración de los Sistemas de Información}

La integración de sistemas de información conlleva mucho más que el uso de una única interfaz para la búsqueda, recuperación y consulta de información. Es necesario realizar una serie de planificación previa que nos permita establecer relaciones de contenido entre los distintos recursos de información existentes en la organización. Hasta hace poco al hablar de integración de sistemas de información se pensaba en la reorganización de documentos para la creación de herramientas comunes para la recuperación de información relacionando los contenidos cuando el usuario iniciaba los procesos de búsqueda y cuando el sistema devolvía los resultados.

Pero en realidad, la integración de sistemas de información para gestión de conocimiento requiere una gestión documental distribuida que permita combinar la gestión documental y el resto de aplicaciones del entorno de trabajo del usuario (Lotus, 1998, p. 5). En definitiva se trata de realizar los procesos de integración de información desde el mismo momento en el que se está introduciendo la información en el sistema (figura 2). Esta integración previa se realiza en tres pasos: 


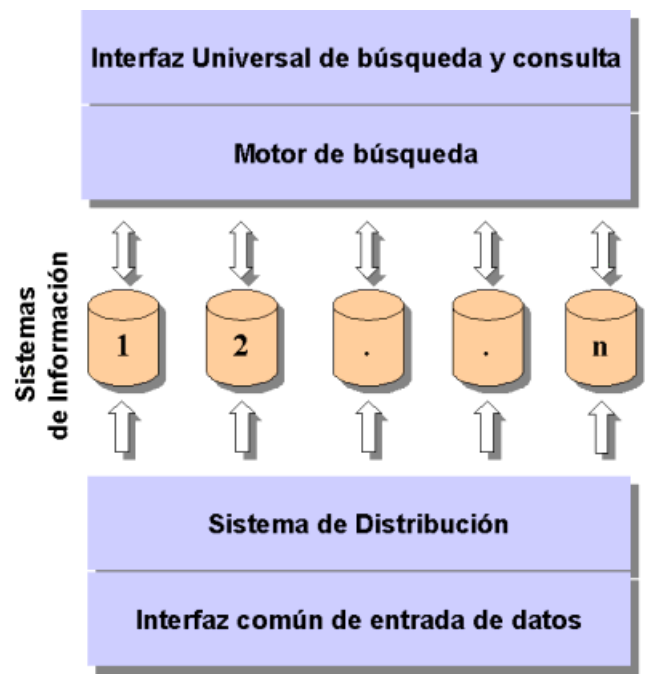

Figura 2: Esquema para la Integración de los Sistemas de Información

1. Estudio y caracterización de los diferentes tipos de información extrayendo contenidos comunes y contenidos específicos.

2. Diseño de herramientas que permitan el mantenimiento de diversos sistemas a partir de una única entrada de información.

3. Estudio y aplicación de los esquemas mentales de conocimiento de los propios usuarios que mantienen el sistema a través de una serie de operaciones de análisis y síntesis del contenido a introducir en el sistema.

La información integrada a partir de los esquemas cognitivos de los usuarios aporta conocimiento a la totalidad de la organización. Por supuesto también son imprescindibles la existencia de las herramientas de búsqueda, recuperación y consulta a partir de una única interfaz de usuario.

\subsection{Groupware = Mensajería + Flujo de Trabajo + Trabajo en Grupo}

Con el término groupware denominamos a aquellas aplicaciones que ofrecen las funcionalidades de mensajería, trabajo en grupo y flujo de trabajo a través de redes informáticas a través de una única interfaz y con la finalidad de integrar recursos informativos y humanos.

La mensajería electrónica no se limita a las relaciones entre usuarios, sino también entre usuarios, procesos y fuentes de información. Es aquí donde la men- 
sajería se relaciona con los procesos de trabajo en grupo y flujo de trabajo. Las alteraciones de contenidos en los recursos del sistema de información son comunicadas a los usuarios asociados de algún modo a dichos recursos. La introducción de nueva información en el sistema puede dar lugar a un informe diario que se envía a través de mensajería electrónica a los usuarios interesados (difusión selectiva de información). La elaboración de nuevas versiones de documentos de proyectos o la modificación de estados en el flujo de trabajo se comunica al personal relacionado con un proyecto o tarea. Así pues, se hace necesaria una integración de sistemas de información para que se lleven a cabo las funciones de mensajería que propone el groupware.

Por su parte, el trabajo en grupo tiene como objetivo cubrir las necesidades de los trabajadores de una organización en cuanto a las herramientas para compartir información y para la elaboración de documentos en colaboración. La elaboración cooperativa de documentos se realiza a partir de foros de debate que permiten el intercambio de impresiones y la retroalimentación en los procesos de adquisición de conocimiento por parte de los usuarios. El trabajo en grupo supone la creación de macroestructuras de información a partir de las microestructuras de los usuarios individuales.

En el trabajo en grupo es imprescindible el control de versiones o estados de los diferentes documentos, en los cuales los diferentes usuarios que tienen acceso a los mismos pueden crear notas y nuevas versiones. Ademáss los niveles de difusión de un documento alertan a estos de los cambios que se producen en los mismos. Ambas funciones, control de versiones y niveles de difusión, constituyen el nexo de unión entre el Trabajo en Grupo y el Flujo de Trabajo. El XML supone un gran avance en la consecución de la elaboración estructurada de documentos, en los que el usuario final se olvida de la estructura y presentación del documento (que son definidos por el gestor del sistema de información) y se centra en el contenido y elementos informativos de los mismos.

Respecto al acceso a la información lo más destacable es la desaparición de los intermediarios. El documentalista o técnico en información ya no es el intermediario, sino que se encarga de diseñar, mantener y actualizar el sistema de información de la empresa. Esto es posible gracias a una política de niveles de acceso a la información:

- Nivel público: Accesible a cualquier usuario pertenezca o no a la organización.

- Nivel corporativo: Todos los miembros de la empresa.

- Nivel de grupo/proyecto de trabajo: El usuario tiene acceso a todos los documentos que han sido creados por los miembros de su grupo/proyecto de trabajo. 
- Nivel de perfil personalizado: Algunos documentos pueden ser utilizados por ciertas personas que no pertenecen a ninguno de los niveles expuestos anteriormente.

- Nivel individual: Solamente el usuario en cuestión tiene acceso a sus documentos personales.

Es evidente que el trabajo en grupo, al permitir el intercambio dinámico de información entre los distintos agentes (humanos o informáticos) contribuye a la obtención de conocimiento a partir de la información en el entorno. Además, la organización de la información documental puede organizarse a partir de un esquema de acceso a los mismos (Pastor y Saorín, 1998). De este modo, un profesor podría introducir sus apuntes, horarios, tutorias y prácticas en un sistema de información que podría ser consultado por los alumnos.

El flujo de trabajo dentro del ámbito de la información documental es una ampliación de la mensajería. En este tipo de procesos se hacen circular los documentos según un esquema preestablecido (Alin, Lafont y Macary 1997, p. 58). Básicamente el Flujo de Trabajo aborda la circulación de la información documental en el seno de una organización a través de una serie de procesos para los que se han determinado unos ciertos plazos de ejecución.

Por lo tanto, hay que definir los procesos con base en la circulación de información y la asignación de agentes a dichos procesos, al tiempo que se definen los ámbitos de difusión de la información documental, a medida que se producen cambios en la misma durante la ejecución de los procesos. Un sistema de flujo de trabajo obliga a una serie de cambios en la mentalidad de los usuarios (en todos sus niveles de organización), así como la implantación de sistemas informáticos de gestión y comunicación de la información (al igual que ocurre con la mensajería electrónica y el trabajo en grupo).

En realidad, el flujo de trabajo no es sino una herramienta para la organización del flujo de información de forma cooperativa. A partir de los estudios de los flujos de trabajo se obtiene información agregada de los recursos de información documental junto con los procesos de las diferentes tareas: es decir, conocimiento de la dinámica informativa de la organización.

No deben confundirse trabajo en grupo y flujo de trabajo. Mientras que el trabajo en grupo se centra en la elaboración colaborativa de información y en el acceso a la misma, las técnicas de flujo de trabajo abordan el análisis de los procesos de la organización y de los recursos de información que intervienen en los mismos. 


\section{Un Modelo para la Gestión del Conocimiento en organizaciones universitarias}

A continuación se propone un modelo cuyo objetivo es la identificación de los procesos, agentes y recursos documentales del sistema de información. El ámbito de aplicación tendría un nivel de generalidad/especialización, adaptado a las necesidades de detalle en la descripción para un determinado proceso o para las relaciones entre la totalidad de la unidades de una organización. Este modelo se basa en el entorno anteriormente descrito y puede ayudar a los miembros de la comunidad universitaria en sus procesos de gestión del conocimiento

\subsection{Elementos del Modelo}

El modelo propuesto tiene como objetivo la identificación de los procesos, agentes y recursos documentales del sistema de información. Contempla los siguientes elementos:

- Procesos: Tareas asignada a un agente cuya finalidad es la gestión o elaboración de información documental, con independencia del soporte de la misma. Los procesos poseen una entrada y una salida de recursos de información.

- Agentes: Realizan los procesos del sistema de información. Los agentes pueden ser los usuarios del sistema o aplicaciones informáticas que realicen de forma automatizada tareas de gestión de la información documental (indización, resúmen, difusión selectiva de información, etc.). Son una parte imprescindible del sistema de información de la empresa.

- Recursos de información documental: Estos elementos constituyen la materia prima del sistema. Es imprescindible tener en cuenta la realidad de una organización, donde se gestionan documentos electrónicos y en papel. Una de las paradojas con la que nos podemos encontrar es que, a pesar de utilizar medios electrónicos para elaborar documentos en papel, en ocasiones los ficheros informáticos no se conservan. La tipología de estos recursos dentro de nuestro modelo es la siguiente:

- Documentos en papel

- Documentos electrónicos

- Bases de datos

- Mensajes electrónicos

\subsection{Herramientas}

El modelo se estructuraría en las siguientes herramientas:

- Definición de procesos: En esta fase se asignaría un nombre al proceso y se identificarían los agentes que los desarrollan y los plazos de finalización. 

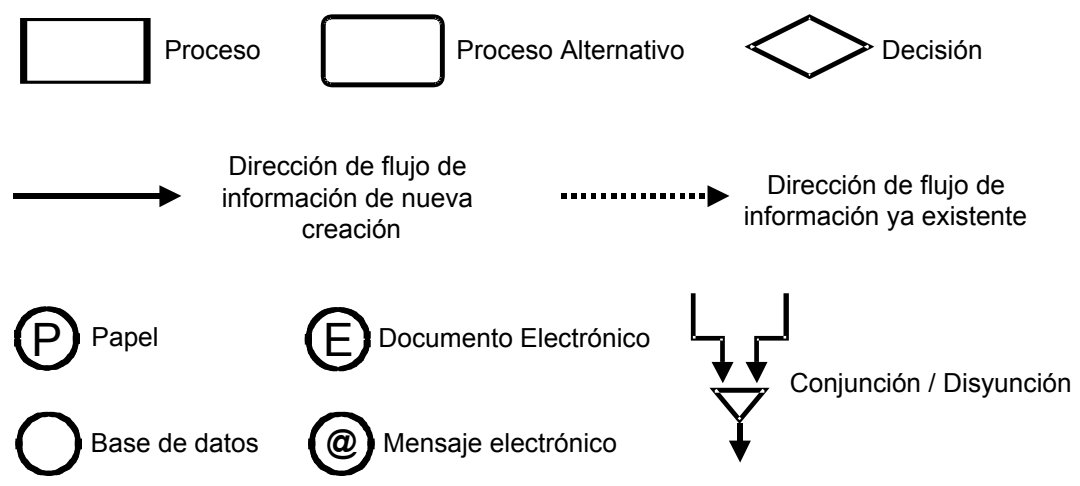

Figura 3: Elementos para la representación gráfica del modelo

Es imprescindible realizar una estructuración lógica del orden de ejecución de los mismos estableciendo los procesos precedentes y consecuentes, los recursos documentales entrantes, salientes, elaborados y/o modificados.

- Definición de agentes: Se asignaría una denominación al agente, al tiempo que se establecen los procesos en los que toma parte, recursos documentales a los que tiene acceso. Se realizará una descripción del ámbito del agente (departamento, entorno de la empresa, etc.). Puede ser de utilidad definir grupos de usuarios y niveles de acceso de los mismos, con lo que la aparición de nuevos recursos y usuarios no obligaría a la modificación del diseño del sistema de información, al tiempo que facilitaría la elaboración y el mantenimiento del diseño del sistema de información.

- Definición de los recursos documentales: Sería en esta fase cuando se describirían los diferente elementos informativos que toman parte en el sistema. Se identificará el tipo de recurso, descripción del mismo (contenido, estructura y presentación) y plazo de vigencia. En el caso de que el tipo de recurso definido sea un mensaje electrónico hay que especificar entre qué agentes va a ser difundido.

- Representación gráfica: Nos hemos basado en la técnica de flujo de trabajo documental propuesto por Hilera y Martínez (Hilera y Martínez, 1998). Dicha técnica se ha adaptado para obtener una simplificación de los procesos y establecer la tipología de los recursos de información. En este modelo los agentes (usuarios y aplicaciones) constituyen columnas separadas entre sí por líneas discontinuas. Dentro de estas columnas se situan las tareas, condiciones de los flujos de trabajo, conjuntores/disyuntores y los recursos informativos (Figura 3). 


\subsection{Fases de Aplicación}

La aplicación de este modelo se realizará en cuatro fases (Figura 4):

1. Fase de Definición: Comprensión y sistematización de la estructura formal y funcional de la organización. En esta fase se identificarán los objetivos hacia los que se orientará el sistema de información de la empresa.

2. Fase de descripción: Estudio y análisis de la información y documentación en las organizaciones; esto es, análisis de la información usada, de la información generada, de los flujos de información internos y externos, de las necesidades de información y de las necesidades de tratamiento documental. Supone la realización de un trabajo descriptivo, desde el punto de vista informativo, de la organización objeto de estudio.

3. Fase de diseño del sistema de información: Esta fase, la fase creativa, supone el intento de reorientar los comportamientos informativos de la organización, de modo global, sectorial o funcional, a partir del estado de situación reflejado en la fase anterior, diseñando el esquema formal que representará al nuevo sistema de información.

4. Fase de desarrollo (implantación del sistema de información): Esta operación es la que hace real y operativo un esquema previamente diseñado. La fase de implantación consiste en llevar a la práctica cada una de las indicaciones manifestadas en el diseño del sistema de información, de

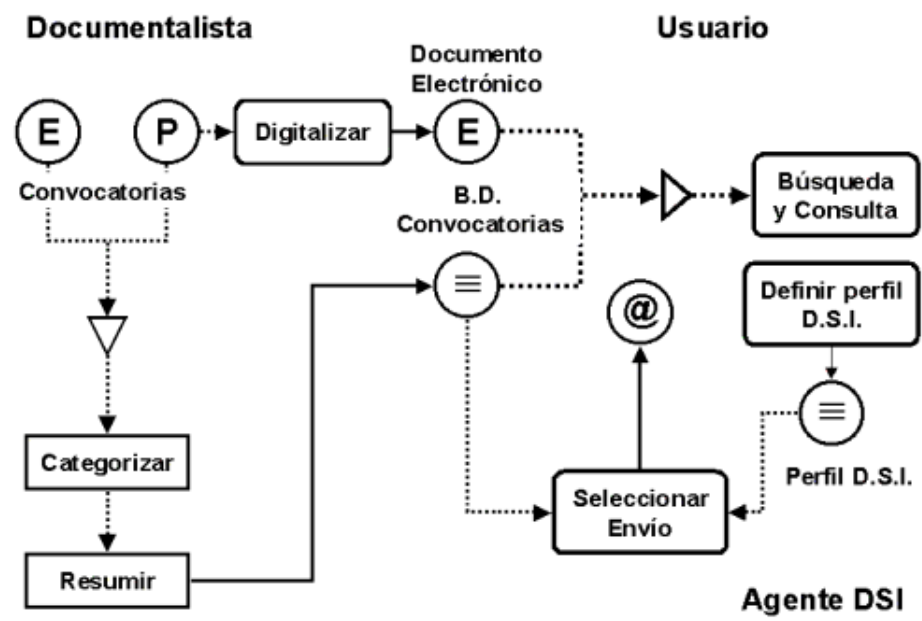

Figura 4. Ejemplo de representación gráfica del modelo 
modo planificado. Será el momento de seleccionar herramientas de desarrollo y asignar agentes concretos a los procesos definidos.

5. Fase de evaluación: En esta fase se identificarán las posibles disfunciones que presente el funcionamiento del sistema. Para ello se recurrirá a la gestión de calidad total (Arias y Portela, 1997), que en realidad debe estar presente en todas las fases del modelo.

\section{Servicios y productos de información para la comunidad universitaria}

\subsection{Atención al Público}

La atención al público es fundamental en la universidad ya que constituye un punto de acercamiento al usuario con una necesidad de información, al tiempo que abre una puerta de acceso a sus recursos de información. La atención puede ser presencial, telefónica, a través del correo electrónico o correo postal. También puede realizarse de una forma activa o pasiva por parte del usuario. En cualquier caso, el personal del servicio de información deberá disponer de información preparada sobre aquellos temas de consulta más frecuentemente.

Será aquí donde los gestores de información podrán poner, a través de herramientas de trabajo en grupo, sus conocimientos paras las consultas de los usuarios. A través de un foro, lista de distribución o biblioteca de referencia se podrá ofrecer un servicio más rápido y de calidad, ya que el profesional dispondrá de una base de conocimiento para la resolución de las posibles dudas que pudieran surgir.

La atención al público incluye la difusión de las actividades del servicio dentro y fuera de la comunidad universitaria. Tampoco hay que olvidar que la gestión de una unidad de estas características conlleva una gestión documental adecuada, convirtiéndose en centros de documentación especializados en temas universitarios. La información documental debe estar correctamente organizada de una manera que permita su rápida localización.

\subsection{Publicaciones: guías y boletines}

Es imprescindible la edición de publicaciones (ya sea en formato papel o electrónico) que ofrezcan información sobre diferentes aspectos de la universidad donde se desarrollen las tareas del servicio de información. Básicamente podemos identificar las siguientes publicaciones:

- Boletín universitario: Esta publicación recogería acuerdos administrativos, convocatorias económicas, becas, proyectos, programas europeos, información de transferencia de investigación, norma académico-administrativa y disposiciones específicas del rectorado. Su difusión debe alcanzar a todos los ámbitos de la comunidad universitaria y a otros instituciones 
sociales (bibliotecas municipales, gobierno regional, etc.). La edición electrónica permite el acceso a esta publicación elaborada a partir de los sistemas de bases de datos del sistema de gestión de información.

- Guía de la universidad: Su objetivo es mostrar de forma clara la Institución a todos los miembros de la misma: profesores, alumnos y personal de administración y servicios. Abarcaría todos los servicios y unidades. Esta publicación también debe recoger las normas de matrícula y los planes de estudios. Esa misma información se puede editar en formato electrónico para su distribución en CD-ROM, disco o a través de Internet.

- Guía multimedia: En la actualidad, las universidades se desenvuelven dentro de una sociedad en donde los elementos visuales, textuales y sonoros se unen para una mayor y mejor comprensión de los contenidos informativos. En esta línea es imprescindible disponer de un producto de calidad y con información completa y actualizada. Esta guía permitiría un salto cualitativo y cuantitativo respecto a la información que es capaz de ofrecer una publicación a través de un único medio, al tiempo que es capaz de integrar la consulta de un CD-ROM y la información puntual y constantemente actualizada en Internet.

- Guía para alumnos de educación secundaria: Destinada a los alumnos de último curso de enseñanzas medias, con el objeto de ayudarles en la medida de lo posible en la difícil tarea de elección de estudio universitario a cursar. Complementariamente a esta publicación deben desarrollarse actividades de visitas a distintos centros de secundaria, manteniendo contacto directo con estos estudiantes y con sus profesores.

- Otras publicaciones: Folletos, campañas de difusión, carteles, etc.

\subsection{EI WEB: La universidad como un portal}

Actualmente los servicios Web no solamente ofrecen información sobre las actividades de investigación, docencia, titulaciones, convocatorias, becas, actividades, cursos, etc. También están ofreciendo servicios de valor añadido. Las nuevas posibilidades de docencia y desarrollo de actividades a distancia está orientando los servidores Web hacia la constitución de portales de información y servicios. La posibilidad de recibir a través del teléfono móvil los resultados de preinscripción o la calificación de una asignatura, además de la consulta de bases de datos y la posibilidad de matricularse o solicitar el acceso a la Universidad a través del Web se realizará a corto plazo.

Scire. $6: 2$ (jul.-dic. 2000) 99-120. 


\subsection{Bases de datos en Internet: Integración de contenidos a través de la difusión selectiva de información}

La integración de sistemas de información constituye un elemento primordial para el establecimiento de los sistemas de gestión del conocimiento. El objeto global de estos sistemas no es otro que ofrecer a los universitarios un medio para la adquisición de ese conocimiento, aprovechando la facilidad de integración de sistemas que nos ofrece el sistema Web e incorporando de forma paulatina la información proveniente de otras aplicaciones de gestión de la propia Intranet para obtener:

- Una gestión distribuida con control de seguridad de una base de datos informativa de carácter general para todos los universitarios.

- Una gestión unificada de las bases de datos corporativas de la universidad.

- Un uso eficaz de un interfaz común (cliente Web) que permita la unificación de las aplicaciones de correo electrónico, navegador Web y gestión de bases de datos.

En definitiva, nuestro objetivo es la gestión de información mediante una estructura de bases de datos documentales. Es necesario definir tres niveles de usuarios: administrador, gestores de datos (personal encargado de la introducción, modificación y eliminación de registros) y usuarios base. El uso de dichas bases de datos deberá realizarse a través de Internet, con un triple objetivo. En primer lugar, para facilitar el acceso de cualquier miembro de la comunidad universitaria. En segundo lugar, para permitir el acceso de cualquier usuario de la red y, en tercer lugar, utilizando el mismo medio de comunicación para gestionar la base de datos desde las diversas unidades y servicios. Todo lo anterior a través de una arquitectura cliente-servidor con una interfaz común que evite el tener que instalar aplicaciones específicas en los clientes.

Una de las ideas más atrayentes de este sistema es la de implantar una difusión selectiva de información. Para ello, los usuarios tendrán que expresar sus necesidades informativas a partir de descriptores, y de forma diaria, el sistema analizará las nuevas referencias introducidas y las contrastará con los perfiles de los usuarios, remitiéndoles de forma automática un correo electrónico indicándoles las novedades de su interés.

\section{Cambios en la gestión del Web}

Realmente la Gestión de los Sistemas de Información a través del Web, están sufriendo un proceso de transformación. Anteriormente lo principal era la elaboración de páginas con contenidos informativos correctos y con una interfaz de usuario lo suficientemente agradable como para atraer a los usuarios y consultar 
dicha información. A los profesionales se les exigía un conocimiento relativamente profundo de lenguaje HTML y el manejo de editores de páginas Web como Frontpage. A partir de una imagen corporativa y mediante el uso de plantillas, se comenzaba a la edición de información bien por la persona encargada en el servicio de información de esta tarea o por la totalidad del personal técnico. El problema fundamental era que se pedía que la página se visualizara correctamente, sin darle importancia a la estructuración del documento.

Además, se utilizaba la página Web como medio para publicar cualquier tipo de información: artículos, listados de personal, texto completo de convocatorias. En definitiva, información ya existente en ficheros electrónicos que había que transformar en páginas HTML. A pesar de que los editores más utilizados en la actualidad permiten la importación de otros ficheros en formato de procesador de texto, se perdía mucho tiempo en la labor de formateado, elaboración de tablas, eliminación de interpretaciones del filtro de exportación que afectaban a la visualización del documento, etc. Como consecuencia, el profesional dedicaba más tiempo a la maquetación de la página que a la estructuración de la misma.

Sin embargo, ya disponemos de herramientas que permiten que el gestor de información pueda optimizar su tiempo de trabajo. Ya no es necesario elaborar documentos Web de gran longitud o crear complejas estructuras de hipertexto. Actualmente los Sistemas de Información en Web complementan las páginas HTML con documentos en formato PDF que permiten una consulta eficaz y una impresión de calidad. Se elaboraran una serie de índices de acceso, pero los documentos de gran tamaño se depositan en los servidores en un formato compacto, cuya descarga posibilita su consulta sin necesidad de estar conectado en red.

Por otro lado, el HTML ha demostrado que no es la panacea universal, que dispone de una serie de limitaciones: principalmente su falta de flexibilidad y la carencia en estructuras avanzadas de descripción de la estructura documental. El SGML constituye una opción muy compleja para el desarrollo de un lenguaje que solucione dicho problema. Sin embargo, con la aparición del XML se han abierto nuevos campos de actuación. El XML permite separar la estructura de los contenidos del documento a partir de la definición de tipos de documento. El principal escollo del XML es que para la consulta de información en Web debe realizarse una traducción a HTML a partir de traductores que definen el formato de presentación de las estructuras de los documentos XML. Para solucionar este problema el W3C elaboró la versión 1.0 de XHTML que amplia las posibilidades de adaptación del HTML al control de la estructura de los documentos y asegura la supervivencia del HTML como una herramienta válida para su uso en los sistemas de información (Martín y Rodríguez, 2000). Además, la elaboración conceptual de los sistemas de información en Web se están utilizando modelos con-

Scire. $6: 2$ (jul.-dic. 2000) 99-120. 


\section{8}

ceptuales de diseño de estructuras en hipertexto a partir de metodologías bien definidas, como el RMM ampliado (Isakowitz, Stohr y Balasubramanian, 1995).

Todo lo anterior se complementa con el uso de Bases de Datos que permiten la elaboración de consultas predefinidas para el diseño de páginas dinámicas actualizadas en todo momento de forma dinámica. Sistemas como Lotus Domino permiten el diseño de aplicaciones de bases de datos documentales para la gestión, consulta y elaboración de páginas dinámicas — todo ello a través del Web- y están suponiendo una revolución, no solamente por la aparición en sí de estas herramientas, sino por el nuevo perfil de documentalista y del gestor de información que interviene en ellos, y que comienzan a estar capacitados para trabajar directamente con estos sistemas sin necesidad de la figura del técnico o administrador informático.

\section{Conclusiones}

La gestión del conocimiento tiene como objetivo el desarrollo de herramientas de gestión de información que permitan a los usuarios intercambiar y crear conocimiento. Para ello resulta necesaria establecer un entorno de desarrollo mediante la creación de una base organizativa y tecnológica. En una institución universitaria resulta necesaria la implantación de un servicio de información universitario que, con un personal suficientemente formado y con una organización del trabajo bien establecida, desarrolle las herramientas expuestas en este trabajo. Además el groupware y la integración de sistemas de información constituyen una base para el modelo presentado en este trabajo. Por supuesto, dicho modelo se trata de una propuesta totalmente abierta y modular, de forma que se pueda adaptar con el paso del tiempo y según las necesidades de la institución universitaria en donde se aplique.

La atención al público es fundamental en este tipo de servicios, ya que es el nexo entre la información y los usuarios, y en gran medida la forma en la que se desarrolla el intercambio de información. El Web constituye el cliente/servidor universal, una puerta de entrada a un modo de nuevos servicios y productos de información, pero teniendo en cuenta que las nuevas tecnologías son un medio y no un fin, cuyo uso (y su coexistencia con formatos tradicionales de información) permitirá la participación de estudiantes, docentes, investigadores y profesionales en la mejora de los servicios y productos de información.

Finalmente, indicar que el continuo cambio en las tecnologías y la propuesta de nuevos modelos está cambiando el diseño de los sistemas de información basados en Web, de forma que se están optimizando los procesos de trabajo y ofreciendo aplicaciones y soluciones que permiten una consulta más eficaz de los contenidos por parte del usuario, al tiempo que se favorece la comunicación e 
intercambio de ideas entre las personas, lo cual es el motor fundamental para la creación de nuevo conocimiento.

\section{Bibliografía}

Alin, F. ; Lafont, D. ; Macary, J. F. (1997) El Proyecto Intranet. Barcelona : Ediciones Gestión 2000, 1997.

Arboníes, A. L. (2000). El conocimiento no se puede gestionar. // Gestión del Conocimiento.com. URL: <http://www.gestiondelconocimiento.com/documentos2/angel/conogest.htm>. Consultado el 2 de Noviembre de 2000.

Arias Coello, A. ; Portela, I. (1997). Sistema de Información y Sistema de Gestión de Calidad: Relación y dependencia en las organizaciones empresariales. // Actas de las III Jornadas de Información y Documentación Empresarial INDOEM'96. Murcia, 1997, p. 189-196.

Bueno Campos, E. (1998). Gestión del Conocimiento, Aprendizaje y Capital Intelectual. Boletín de Información no 1 del Club Intelect (Diciembre de 1998 / Enero del 1999). URL: <http://www.gestiondelconocimiento.com/documentos2/eduardo_bueno/ aprende.htm>. Consultado el 10 de Octubre de 2000.

Currás, E. (1995). Concierto y desconcierto en la organización del conocimiento actual y su intersección con el mundo de la información. // Scire. 1 : 1 (enero-junio 1995) 328.

Davenport, T. (1996). Knowledge Management: Case Study, Knowledge Management at Hewlett-Packard, Early 1996. Austin : Universidad de Texas. URL: <http://www.bus.utexas.edu/kman/hpcase.htm>. Consultado el 27 de Septiembre de 2000.

García González, F. (1998). La Universidad del Siglo XXI como un modelo de industria de la Información y del Conocimiento. // División de Productos Informativos Electrónicos, DPI, Centro de Gestión de Información, Universidad de Camagüey (Cuba), 1998. URL=<http://www.gestiondelconocimiento.com/documentos2/ fidel_garcia/resumen_modelo.htm>. Consultado el 17 de Octubre de 2000.

Hilera González, J. R. ; Martínez Sánchez, J. M. (1998). Ingeniería Documental asistida por Computador (CADE). // Actas de las VI Jornadas Españolas de Documentación FESABID 98. Edición en CD-ROM. Valencia, 1998.

Isakowitz, T. ; Stohr, E. ; Balasubramania, P. (1995). A Methodology for the Design of Structures Hipermedia Aplication. // Comunications of the ACM 38:8 (agosto 1995) 34-44.

LOTUS (1998). Libro blanco Lotus Domino.doc. Lotus Development Corporation, 1998.

Martín Galán, B.; Rodríguez Mateos, D. (2000). Estructuración de la información mediante XML: un nuevo reto para la gestión documental. // Actas de las VII Jornadas Españolas de Documentación FESABID 2000. Edición en CD-ROM, Valencia, 113123.

Martínez Méndez, F. J. (1997). Ingenieros de la Información. // Actas de las III Jornadas de Información y Documentación Empresarial INDOEM’96. Murcia, 1997, p. 25-31.

Scire. $6: 2$ (jul.-dic. 2000) 99-120. 
Martínez Méndez, F. J. (1999). El salto desde la Gestión de Información a la Gestión del Conocimiento. // Scire 5 : 1 (enero-junio 1999) 41-54.

Pastor Sánchez, J. A. (1996). El nuevo documento electrónico de la tabla relacional al hiperdocumento. // Scire. 2 : 2 (julio-diciembre 1996) 139-151.

Pastor Sánchez, J. A.; Saorín Pérez, T. (1998). La Escritura Hipermedia. // Cuadernos de Documentación Multimedia. 6-7 (1998) 221-238.

Rodríguez de las Heras, A. (1991) Navegar por la información. Madrid: Fundesco, 1991.

Saorín Pérez, T. (1997). Ofimática Documental. // Scire. 3 : 2 (1997) 55-72.

Sebastiá, M. (1992). De la invisibilidad a la visibilidad. Reflexiones sobre la formación de especialistas y usuarios de las Tecnologías de la Información. // Revista Española de Documentación Científica 15 : 1 (1992) 31-43. 